\title{
A new method for calculating the combined anchor-concrete support of underground structures
}

\author{
Mikhail Pleshko ${ }^{1, *}$, Besarion Meskhi ${ }^{2}$, and Marianna Pleshko ${ }^{3}$ \\ ${ }^{1}$ National University of Science and Technology MISIS (Moscow Institute of Steel and Alloys), \\ Leninskij Av., 4, Moskva, 119991, Russia \\ ${ }^{2}$ Don State Technical University, pl. Gagarina, 1, Rostov-on-Don, 344022, Russia \\ ${ }^{3}$ Rostov State Transport University, Rostovskogo Strelkovogo polka sq., 2, Rostov-on-Don, 344038, \\ Russia
}

\begin{abstract}
The problematic aspect of the underground structures construction in the urban area is the negative impact of construction on neighboring buildings and structures. To exclude emergency situations, it is required to control the interaction of the support of the underground structure and the surrounding soil mass by including additional regulatory elements, such as anchors, in the system. The article presents a new method for calculating the anchor-concrete support of underground structures and the results of monitoring of tensile forces in the bolting. It has been established that the tensile force has the highest constant value at the well mouth - jointing of the anchor rod, and then decrease exponentially.
\end{abstract}

\section{Introduction}

The development of the transport system in large cities generates a need for the comprehensive development of the underground space. The transfer of traffic flows to the underground level allows to solve a number of key tasks of the city development: to increase the speed of traffic and the volume of passenger and freight traffic; improve traffic safety; improve the ecological situation and reduce the technospheric detrimental effect on the surrounding buildings [1-2].

The problematic aspect of underground structures construction in the urban area is the negative impact of construction on neighboring buildings and structures. It can be evident as excessive soil and foundation settlement, caving etc. Avoiding accidents requires the management actions focused on reciprocal influence between support frames of underground structures and the surrounding soil mass system by incorporating additional regulatory elements. One of these elements is the bolting, which is becoming increasingly common in urban construction practice. At the same time, some theoretical aspects of calculating the joint usage of bolting and concrete support remain undeveloped in the frame of underground structures. [2-4].

*Corresponding author: mixail-stepan@mail.ru 


\section{Materials and Methods}

Analytical methods for calculating the support of underground structures are based on the provisions of the elasticity theory and continuum mechanics used in this study. To assess the reliability of the obtained results, in-situ studies were also carried out to determine the tensile forces in the anchors by means of strain gauges glued along the length of the bolt shaft. Anchors with strain gauges were installed in a vertical shaft during the construction. [5-6].

\section{Results}

In accordance with the concepts of continuum mechanics and the mechanics of underground structures, when examining the anchor-concrete support of vertical shafts, it is possible to proceed to the calculation model shown in Fig. 1.

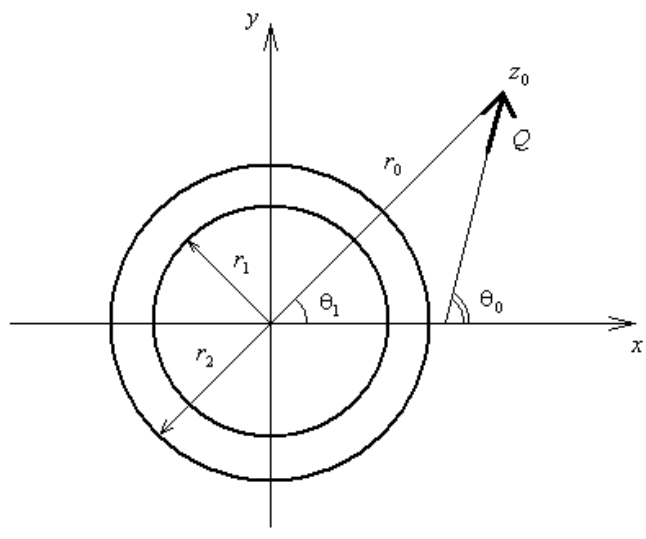

Fig. 1. Calculation model.

At an arbitrary point $z_{0}$ area $|z| \geq r_{1}$, which has coordinates $\left(r_{0}, \theta_{0}\right)$, at an angle $\theta_{1}$ to the real axis we apply point force $Q$. It is caused by the appearance of the anchor tension and is determined with account for the work method. Let us consider the algorithm for determining the offset in the system. From the theory of elasticity, functions of complex potentials $\varphi$ and $\psi$, characterizing the stress-strain state of an infinite plane from the action of a point force. In our case, these potentials have the following form

$$
\begin{aligned}
& \varphi(z)=-\frac{X+i Y}{2 \pi(\chi+1)} \ln \left(z-z_{0}\right)+\varphi_{0}(z), \\
& \psi(z)=\chi \frac{X+i Y}{2 \pi(\chi+1)} \ln \left(z-z_{0}\right)+\frac{X+i Y}{2 \pi(\chi+1)} \cdot \frac{\bar{z}_{0}}{z-z_{0}}+\psi_{0}(z),
\end{aligned}
$$

when $X, Y$ - force projection $Q$ on real and imaginary axis respectively; $\chi=3-4 v$.

Potentials $\varphi_{0}(z)$ and $\psi_{0}(z)$, additively in the expressions (1) determined depending on the additional conditions.

In the case of the action of a point force in an infinite plane weakened by a circular hole, the complex potentials $\varphi$ and $\psi$ take the form 


$$
\begin{aligned}
\varphi(\xi) & =A(\chi) \ln \left(\xi-\xi_{0}\right)-\bar{B}(\chi) \ln \left(1-\frac{1}{\bar{\xi}_{0} \xi}\right)-\bar{A}(\chi) \frac{1-\rho_{0}^{2}}{\bar{\xi}_{0}^{2}\left(1-\bar{\xi}_{0} \xi\right)} \\
\psi(\xi) & =B(\chi) \ln \left(\xi-\xi_{0}\right)-A(\chi)\left[\frac{\bar{\xi}_{0}}{\xi-\xi_{0}}-\frac{1}{\xi_{0} \xi}\right]-\bar{B}(\chi)\left[\frac{\bar{\xi}_{0}}{\xi\left(1-\xi_{0} \xi\right)}-\frac{1}{\xi^{2}}\right]- \\
& -\bar{A}(\chi)\left[\ln \left(1-\frac{1}{\bar{\xi}_{0} \xi}\right)+\frac{1-\rho_{0}^{2}}{\bar{\xi}_{0} \xi\left(1-\bar{\xi}_{0} \xi\right)^{2}}\right]
\end{aligned}
$$

when

$$
\begin{aligned}
& \xi=\frac{z}{r_{1}}, \\
& A(\chi)=-\frac{Q \exp \left(i \theta_{1}\right)}{2 \pi(\chi+1)}, \\
& B(\chi)=\chi \frac{Q \exp \left(-i \theta_{1}\right)}{2 \pi(\chi+1)}, \\
& Q=(X+i Y) \exp \left(-i \theta_{1}\right)=(X-i Y) \exp \left(i \theta_{1}\right), \\
& r_{1}-\text { hole radius. }
\end{aligned}
$$

In the case of bolt shaft radial installation force direction $Q$ is normally correlated to the hole contour, consequently $\theta_{1}=\theta_{0}$. For a variable $\xi$, the contour of the hole is represented by a unit circle $|\xi|=1$, and the interface between two sets by a circle $|\xi|=r$, $r=\frac{r_{2}}{r_{1}}$

We introduce a new variable $\zeta=\frac{\xi}{r}$, hereby determining the conformal mapping of the area $|\xi| \geq 1$ to the area $|\zeta| \geq \frac{1}{r}$, and the points of the outer contour of the circle $1 \leq|\xi| \leq r$ (points of the interface between two sets) are mapped onto points of the unit circle $|\zeta|=1$.

For a new variable, the complex potentials take the following form

$$
\begin{aligned}
\varphi(\zeta)= & A(\chi) \ln \left(r\left(\zeta-\zeta_{0}\right)\right)-B(\chi) \ln \left(1-\frac{1}{r^{2} \zeta_{0} \zeta}\right)-\bar{A}(\chi) \frac{1-r^{2} \rho_{0}^{2}}{r^{2} \zeta_{0}^{2}\left(1-r^{2} \zeta_{0} \zeta\right)} ; \\
\psi(\zeta)= & B(\chi) \ln \left(r\left(\zeta-\zeta_{0}\right)\right)-A(\chi)\left[\frac{\zeta_{0}}{\zeta-\zeta_{0}}-\frac{1}{r^{2} \zeta_{0} \zeta}\right]-\overline{-}(\chi)\left[\frac{\zeta_{0}}{\zeta\left(1-r^{2} \zeta_{0} \zeta\right)}-\frac{1}{r^{2} \zeta^{2}}\right]- \\
& -\bar{A}(\chi)\left[\ln \left(1-\frac{1}{r^{2} \zeta_{0} \zeta}\right)+\frac{1-r^{2} \rho_{0}^{2}}{r^{2} \zeta_{0} \zeta\left(1-r^{2} \zeta_{0} \zeta\right)^{2}}\right] .
\end{aligned}
$$

The displacement vector is expressed in terms of complex potentials as follows

$$
u(\chi, \mu, \zeta)=\frac{1}{2 \mu}\left[\chi \varphi(\zeta)-\xi \overline{\varphi^{\prime}(\zeta)}-\overline{\psi(\zeta)}\right]
$$


To determine the displacement field in the entire area $|\zeta| \geq \frac{1}{r}$, let us set the displacement function in the form

$$
U(\zeta)= \begin{cases}u\left(\chi_{1}, \mu_{1}, \zeta\right)+u_{1}(\zeta), & \frac{1}{r} \leq|\zeta| \leq 1, \\ u\left(\chi_{2}, \mu_{2}, \zeta\right)+u_{2}(\zeta), & |\zeta| \geq 1,\end{cases}
$$

when $\quad u_{1}(\zeta)$ - holomorphic function in a circle $\frac{1}{r} \leq|\zeta| \leq 1$,

$u_{2}(\zeta)$ - holomorphic function in the externality of the unit circle $|\zeta| \geq 1$, notably

$u_{1}(\zeta)=0$ in area $|\zeta| \geq 1, u_{2}(\zeta)=0$ in area $\frac{1}{r} \leq|\zeta| \leq 1$.

For $E_{1}=E_{0}, v_{1}=v_{0}$ functions $u_{1}(\zeta), u_{2}(\zeta)$ equal to zero.

The condition of continuity of displacements at the interface between two sets $|\zeta|=1$ has the form

$$
u\left(\chi_{1}, \mu_{1}, \zeta\right)+u_{1}(\zeta)=u\left(\chi_{2}, \mu_{2}, \zeta\right)+u_{2}(\zeta)
$$

On the basis of the above dependencies, the components of the stress-strain state of a monolithic concrete support or soil mass at a particular point can then be determined.

To evaluate the performance of reinforced concrete bolting in vertical shafts, a number of in-situ studies were performed, which made it possible in particular to determine the regularities of the effect of the jointing of the anchor in the shaft on the distribution of forces along its length.

Fig. 2 shows a typical picture of the distribution of tensile forces along the length of the anchor at different sizes of jointing. The minimum tensile forces in the anchors are observed when the bolt shaft is fully embedded in the borehole $\left(l_{3}=1,8 \mathrm{~m}\right)$, at $l_{3}=1,4 \mathrm{~m}$ the maximum force is greater by an average of 1,31 times, at $l_{3}=1,0 \mathrm{~m}-1,72$ times.

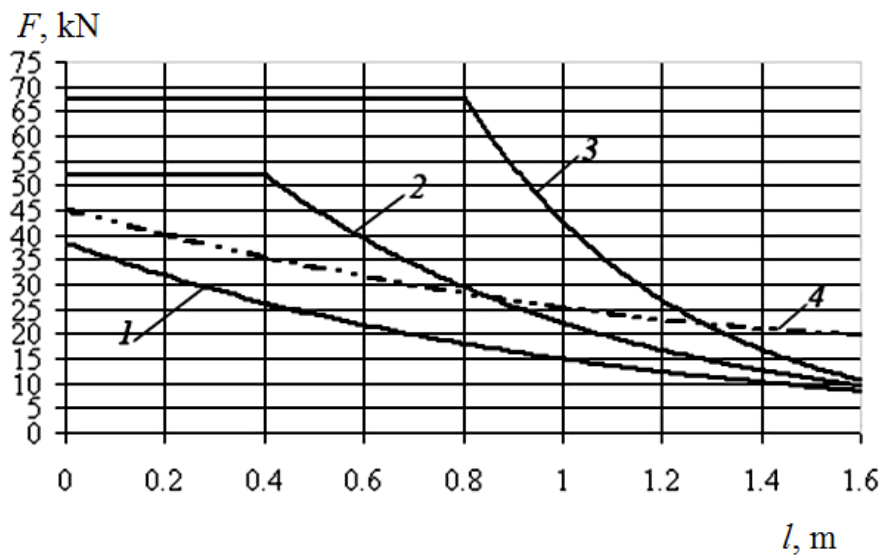

Fig. 2. Distribution of tensile forces in a reinforced concrete anchor

1 - at $l_{3}=1,8 \mathrm{~m} ; 2$ - at $l_{3}=1,4 \mathrm{~m} ; 3$ - at $l_{3}=1,0 \mathrm{~m} ; 4$ - curve of calculated values, obtained as a result of mathematical modeling for complete jointing of the anchor in the shaft. 


\section{Conclusions}

The analysis of the results shows that the magnitude of the forces does not significantly affect the character of their distribution along the length of the anchor, which is similar for all experimental sections. Tensile forces have the largest constant value at the well mouth jointing of the anchor rod, and then decrease exponentially.

The comparison of the experimental and calculated models indicates an identical qualitative picture of the distribution of effort along the length of the anchor when the bolt shaft is completely embedded. The deviation of the maximum effort in the vicinity of the anchor's mouth for the three test sites does not exceed $19 \%$.

It can therefore be concluded that the best working capacity is possessed by reinforced concrete anchors with complete bolt shaft embedment, in which the lowest intensity of tensile forces is observed. With a decrease in the length of the embedment, a considerable increase in the tensile forces in the anchors occurs, which leads to a reduction in the application and durability of the structures. In addition, anchors with full borehole filling have better corrosion resistance.

\section{References}

1. M. D. Molev, ARPN Journal of Engineering and Applied Sciences, 10(16) (2015).

2. M.S. Pleshko, O.V. Pashkova, Gornyj zhurnal, 3 (2015).

3. M. Pleshko, I. Voinov, A. Revyakin MATEC Web of Conferences series, 106, 05004 (2017).

4. B. Meskhi, M. Pleshko, Y. Buligin, et.al. IOP Conference Series: Earth and Environmental Science, 90, 012217 (2017)

5. F.I. Yagodkin, A.Y. Prokopov, M.S. Pleshko, A.N. Pankratenko IOP Conference Series: Earth and Environmental Science Series. "Innovations and Prospects of Development of Mining Machinery and Electrical Engineering - Transportation of Mineral Resources", 062014 (2017).

6. D, Shuxue, J. Hongwen, C. Kunfu,. International Journal of Mining Science and Technology 27 (2017). 